\title{
Calibração do "simplified simple biosphere model - SSiB" para áreas de pastagem e floresta na Amazônia com dados do LBA.
}

\author{
Francis Wagner Silva CORREIA ${ }^{1}$; Regina Célia dos Santos ALVALÁ $;$ Antônio Ocimar MANZI ${ }^{3}$; Ralf \\ GIELOW $^{2}$; Paulo Yoshio KUBOTA ${ }^{1}$
}

\begin{abstract}
RESUMO
Os parâmetros do "Simplified Simple Biosphere Model"-SSiB foram validados e posteriormente calibrados para os sítios de

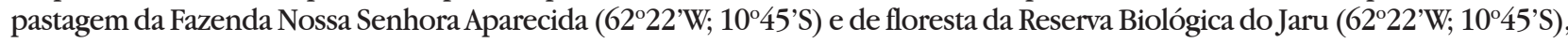
ambos situados no estado de Rondônia. Foram utilizadas medidas micrometeorológicas e hidrológicas obtidas durante o período seco de 2001, como parte do Experimento de Grande Escala da Biosfera-Atmosfera na Amazônia - LBA. Os resultados indicam que o modelo simulou bem o saldo de radiação, tanto na pastagem quanto na floresta. O fluxo de calor latente foi superestimado nos dois sítios nos períodos de simulação, o que deve estar relacionado aos parâmetros utilizados no cálculo dessa variável. O modelo subestimou o fluxo de calor sensível na pastagem e na floresta, principalmente no período noturno; porém, para a floresta, os valores foram mais próximos daqueles observados. Com os parâmetros ajustados, melhores estimativas dos fluxos de calor latente e de calor sensível foram geradas e, conseqüentemente, representou melhor as partições de energia na floresta e na pastagem.
\end{abstract}

\section{PALAVRAS-CHAVE}

calibração, SSiB, pastagem, floresta de Terra Firme, Amazônia, LBA.

\section{Calibration of the simplified simple biosphere model (SSiB) for Amazonian pasture and forest sites using LBA data.}

\begin{abstract}
The parameters of the Simplified Simple Biosphere Model - SSiB were validated and subsequently calibrated for the Fazenda

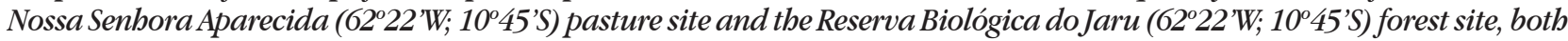
located in the state of Rondônia. Micrometeorological and hydrological data collected during the dry period of 2001, as part of the Large-Scale Biosphere-Atmosphere Experiment in Amazonia-LBA, were used. The results showed that the model simulated well the net radiation, both at the pasture and at the forest. The latent heat flux was super-estimated in both sites. The model subestimated the sensible heat flux at the pasture and at the forest, mainly during the night period; notwithstanding, the values for the forest were nearer to the observed ones. With the calibrated parameters, the model generated better estimations of the latent and the sensible beat fluxes, thus better representing the energy partition both at the forest and at the pasture.
\end{abstract}

\section{KEYWORDS}

calibration, SSiB, "Terra Firme" forest, pasture, Amazon, LBA.

\footnotetext{
${ }^{1}$ Centro de Previsão de Tempo e Estudos Climáticos - Instituto Nacional de Pesquisas Espaciais - CPTEC/INPE. Rodovia Presidente Dutra, Km 040, CEP: 12630-000, Cachoeira Paulista, SP.

${ }^{2}$ Centro de Previsão de Tempo e Estudos Climáticos - Instituto Nacional de Pesquisas Espaciais - CPTEC/INPE. Av dos Astronautas, 1758, Jd. da Granja. CEP: 12227. 010, São José dos Campos, SP.

${ }^{3}$ Escritório Central do projeto LBAno Instituto Nacional de Pesquisas da Amazônia - INPA. Av. AndréAraújo, 2936, Bairro Petrópolis, Caixa Postal 478, CEP: 69011-970 Manaus, AM
} 


\section{ACTA \\ AMAZONICA}

CALIBRAÇÃO DO "SIMPLIFIED SIMPLE BIOSPHERE MODEL - SSIB"

PARA ÁREAS DE PASTAGEM E FLORESTA NA AMAZÔNIA COM DADOS DO LBA.

\section{INTRODUÇÃO}

Desde os trabalhos pioneiros de Charney (1975), diversos experimentos têm mostrado que variações nas características da superfície continental têm significantes impactos no clima. A atmosfera é sensível ao albedo da superfície, à umidade do solo, à rugosidade, e a outras características da vegetação e do solo em diferentes escalas de tempo (Bonan et al. 1992; Foley et al. 1994; Manzi \& Planton, 1996; Costa \& Foley, 2000). Para entender essas interações, não somente qualitativamente, mas também quantitativamente, faz-se necessário utilizar parametrizações da superfície continental (LSPs) que melhor representem a interação superfície-atmosfera. Nas últimas décadas, considerável progresso no entendimento da micrometeorologia tem sido alcançado através de trabalhos teóricos e observações de experimentos de campo (Dolman et al. 1991). Os resultados desses estudos foram incorporados em esquemas de transferência solo-planta-atmosfera (SVATs), sendo acoplados em modelos de circulação geral - MCGs e modelos de mesoescala (Dickinson et al. 1986; Sellers et al. 1986; Sato et al. 1989; Xue et al. 1991; Manzi \& Planton, 1994; Xiu \& Pleim, 2001, entre outros). Vários experimentos numéricos com diferentes esquemas de superfície, na versão "off-line" (desacoplada do MCG), têm sido realizados com a finalidade de validação e estudos de sensibilidade (Sellers, 1985; Sellers \& Dorman, 1987; Sellers et al. 1989; Flerchinger et al. 1998; Delire \& Foley, 1999; Dirmeyer et al. 2000; Nagai, 2002 e 2003). A versão simplificada do "Simple Biosphere Model SSiB" (Xue et al. 1991) foi concebido para simular a interação entre a superfície e a atmosfera, descrevendo os processos de transferência radiativa, turbulenta e controle biofísico da evapotranspiração. Em comparação com outros esquemas de superfície, o modelo SSiB é mais complexo no tratamento do albedo, da energia da superfície e da umidade do solo, e necessita mais parâmetros de entrada. Muitos desses parâmetros podem apresentar incertezas quanto às suas especificações ou não ter nenhuma medida de campo associada aos mesmos. Neste caso, faz-se necessário que os tais parâmetros sejam ajustados ou calibrados, a fim de melhor representar as características físicas e fisiológicas da superfície continental. Um método iterativo de otimização foi utilizado por Sellers et al. (1989) para definir um novo conjunto de parâmetros físicos e fisiológicos da vegetação, que resultou num melhor ajuste entre os fluxos de calor latente e calor sensível calculados e os observados. Medidas micrometeorológicas, incluindo fluxos de calor, coletados acima do dossel da floresta amazônica foram usadas para validar e calibrar os parâmetros do "Simple Biosphere Model -SiB" (Sellers et al. 1989). O conjunto de parâmetros otimizados gerou menores valores de condutância do dossel em relação àqueles descritos na literatura, e mostrou que a resposta estomática ao déficit de pressão de vapor d'água e ao potencial d'água na folha desempenha papel importante na determinação do curso diurno da transpiração da floresta tropical. Rocha et al. (1996) utilizaram dados meteorológicos coletados em dois sítios de pastagem durante o projeto "AngloBrazilian Amazonian Climate Observation Study - ABRACOS”
(Gash et al. 1996), com o propósito de calibrar os parâmetros do modelo $\mathrm{SiB}$ para esse bioma. Os resultados mostraram que o conjunto de parâmetros iniciais superestimou o fluxo de calor sensível, e subestimou a evapotranspiração, enquanto que os parâmetros otimizados melhoraram consideravelmente a estimativa e o ajuste em relação aos fluxos observados. Considerando que a prescrição incorreta ou insuficiente na representação dos parâmetros físicos e fisiológicos da superfície pode conduzir a uma deficiência na representação dos processos de transferência turbulenta entre a superfície e a atmosfera, o que afetaria a previsão climática, o objetivo desse trabalho é calibrar os parâmetros do modelo SSiB para os sítios de pastagem e de floresta de Terra Firme no Estado de Rondônia, utilizando medidas micrometeorológicas e fluxos de superfície obtidos por uma estação meteorológica automática, assim como dados de umidade do solo medidos por sondas de nêutrons.

\section{MATERIAL E MÉTODOS}

Os sítios experimentais (pastagem e floresta) onde foram realizadas as medidas meteorológicas e de fluxos de superfície localizam-se na Fazenda Nossa Senhora Aparecida (62 $22^{\prime} \mathrm{W}$; $\left.10^{\circ} 45^{\prime} \mathrm{S}\right)$ e na Reserva Biológica do Jaru (61⒌ $\left.6^{\prime} \mathrm{W}, 10^{\circ} 46^{\prime} \mathrm{S}\right)$, respectivamente, ambas no Estado de Rondônia.

O sítio de pastagem, situado a $50 \mathrm{~km}$ a nordeste de JiParaná e a 293 m acima do nível do mar, foi estabelecido na década de 80 como área de criação de gado, tendo-se nele instalada uma estação meteorológica automática em 1991. O sítio foi desflorestado em 1981 e está localizado no centro de uma área de aproximadamente $50 \mathrm{~km}$ de raio, onde a vegetação é do tipo gramínea [Brachiaria brizantha]. Maiores detalhes sobre o sítio podem ser encontrados em Culf et al. (1995) e McWilliam et al. (1996). Dados meteorológicos de precipitação pluvial, temperatura do ar, umidade relativa do ar, velocidade e direção do vento e radiação solar global foram registrados pela estação meteorológica automática instalada no topo de uma torre de alumínio de $5 \mathrm{~m}$ de altura. Para medidas de radiação solar utilizaram-se solarímetros (Kipp \& Zonen, Holanda). Termômetros de resistência e um higrômetro (HMP35A-Vaisala, Finlândia) foram usados para medir a temperatura e a umidade relativa do ar, respectivamente. Para medidas de velocidade do vento utilizou-se um anemômetro (Vector A100R, Reino Unido). A precipitação foi medida por um pluviômetro de báscula (Didcot Instrument, Reino Unido). Além disso, medidas da radiação de onda longa incidente e emitida pela superfície foram obtidas por um saldo radiômetro (CNR1 - Kipp \& Zonen, Holanda). Fluxos de calor sensível e de calor latente foram medidos com instrumentos de resposta rápida (Anemômetro sônico GILL, Reino Unido e IRGA LI-6262, LI-COR, Estados Unidos, respectivamente), usando o método de covariância de vórtices turbulentos ("eddy correlation"). A umidade do solo foi medida semanalmente em 13 profundidades $(0,10,0,20,0,40, \ldots$, 2,60 m) utilizando a técnica de sonda de nêutrons (Modelo IH, Didcot Instrument, Reino Unido). 


\section{ACTA \\ AMAZONICA}

CALIBRAÇÃO DO "SIMPLIFIED SIMPLE BIOSPHERE MODEL - SSIB"

PARA ÁREAS DE PASTAGEM E FLORESTA NA AMAZÔNIA COM DADOS DO LBA.
A Reserva Biológica do Jaru, sítio de floresta pertencente ao Instituto Brasileiro do Meio Ambiente e dos Recursos Naturais Renováveis - IBAMA, está situada aproximadamente a $80 \mathrm{~km}$ ao norte de Ji-Paraná e a $120 \mathrm{~m}$ acima do nível do mar. Uma torre meteorológica de alumínio, de $60 \mathrm{~m}$ de altura, foi instalada e instrumentada em 1999. As árvores mais altas próximas da torre são das espécies Cedrella odorata, Inga sp, Dioclea cf bicolor Rbt., Strychnos amazonicus Krukoff, Protium polybotruim e Leonia glycicarpa Ruiz. Informações mais detalhadas desse sítio podem ser encontradas em Gash et al. (1996). Uma estação meteorológica automática foi montada no topo da torre, para medidas de precipitação pluvial, temperatura do ar, umidade relativa do ar, velocidade e direção do vento, radiação solar incidente e de radiação de ondas longas emitida pela atmosfera e pela superfície, com médias coletadas a cada meia hora. Instrumentos semelhantes àqueles descritos para o sítio de pastagem foram utilizados na área de floresta para medir as variáveis meteorológicas e os fluxos de superfície (calor latente e calor sensível). Os dois sítios experimentais considerados neste estudo fazem parte do conjunto de sítios de coleta de dados de superfície do Projeto LBA (ver mapa em http:// lba.cptec.inpe.br/lba/).

\section{PROCEDIMENTO DE CALIBRAÇÃO}

A versão "off-line" do Simplified Simple Biosphere Model (SSiB) foi usada para derivar um conjunto de parâmetros que melhor descrevam os dados experimentais com relação ao período em que foram coletados. Nessa versão, o modelo SSiB é desacoplado do modelo atmosférico e forçado com variáveis micrometeorológicas medidas tanto na área de pastagem (Fazenda Nossa Senhora Aparecida), quanto na de floresta (Reserva Biológica do Jaru). Um período total de 15 dias (dias 1, 4, 6 a 11, 18, 20 e 31 de agosto; 6, 17 a 19 de setembro de 2001) foi selecionado para a calibração na pastagem e outro de 14 dias (1, 5 a 8, 10, 11, 12, 14, 18, 20, 23 de agosto; 18 e 19 de setembro de 2001) para a floresta. O modelo foi integrado a cada 30 minutos forçado com dados de radiação solar incidente $\left(\mathrm{W} \mathrm{m}^{-2}\right)$, radiação de ondas longas da atmosfera (W $\left.\mathrm{m}^{-2}\right)$, precipitação pluvial $(\mathrm{mm})$, temperatura do ar $(\mathrm{K})$, velocidade do vento $\left(\mathrm{m} \mathrm{s}^{-1}\right)$, pressão atmosférica (mb), e umidade específica do ar $\left(\mathrm{g} \mathrm{kg}^{-1}\right)$. A descrição das principais equações do modelo é dada no Apêndice. Os parâmetros iniciais para o sítio de pastagem foram obtidos de Rocha et al. (1996), enquanto para a floresta os parâmetros iniciais foram obtidos da literatura, conforme descrito adiante. O procedimento de calibração minimiza a diferença entre os fluxos calculados e os observados, a partir da otimização dos parâmetros que apresentam maiores incertezas ou nenhuma medida de campo associada. Sellers et al. (1989) e Rocha et al. (1996) descrevem detalhadamente o método iterativo de otimização, cujos passos principais de calibração são apresentados a seguir: (a) Parâmetros medidos in situ ou provenientes da literatura são utilizados para inicializar o modelo. O modelo SSiB-offline é integrado usando dados meteorológicos, como citado anteriormente, para calcular os fluxos de superfície.

(b) A avaliação da qualidade da simulação é feita considerando a diferença entre a fração de evaporação calculada e aquela observada (fluxo de calor latente dividido pela soma do fluxo de calor latente mais o fluxo de calor sensível), ponderada pelo valor absoluto do fluxo de calor latente, como seja:

$F_{i}=\left\{\left(\frac{\lambda E_{i}}{\lambda E_{i}+H_{i}}\right)_{C}-\left(\frac{\lambda E_{i}}{\lambda E_{I}+H_{i}}\right)_{O}\right\} x\left|\lambda E_{i}\right|_{O}$

em que $\mathrm{F}_{\mathrm{i}}$ é o desvio calculado a cada passo de tempo, $\mathrm{lE}_{\mathrm{i}}$ e $\mathrm{H}_{\mathrm{i}}$ são os fluxos de calor latente e sensível, respectivamente, também para cada passo de tempo. Os índices "c" e "o" correspondem aos valores calculados e observados, respectivamente. $\mathrm{O}$ uso da fração de evaporação como um discriminador assegura a estabilidade da otimização, quando não ocorre o fechamento do balanço de energia utilizando dados experimentais.

c) Os desvios em cada passo de tempo são usados para calcular o desvio médio do erro:

$$
\bar{F}=\left(\frac{1}{N} \sum_{1}^{N} F_{i}^{2}\right)
$$

sendo $\mathrm{N}$ o número de medidas e $\bar{F}$ pode ser considerado equivalente ao desvio médio no fluxo de calor latente calculado. Em seguida utiliza-se o algoritmo de minimização de mínimos quadrados, ZXSSQ (IMSL, 1984), para determinar numericamente as derivadas parciais de cada desvio, em relação aos parâmetros otimizados. Os parâmetros são alterados com a finalidade de reduzir $\mathrm{F}_{\mathrm{i}}$. Quando $\mathrm{F}_{\mathrm{i}}$ não pode ser mais reduzido, ou quando as derivadas parciais de $F_{i}$ caem abaixo de um valor especificado, finaliza-se a otimização, gerando um conjunto de parâmetros que fornecem o melhor ajuste.

O diagrama da Figura 1 apresenta as etapas do processo de calibração.

As variáveis prognósticas do modelo foram inicializadas com dados medidos em cada um dos sítios. O armazenamento de água interceptada pelas folhas foi inicializado com valor nulo. Os valores iniciais de umidade do solo foram obtidos após o modelo atingir o equilíbrio. Nos dois sítios o modelo foi integrado por um ano (2001), utilizando medidas de sonda de nêutrons como condição inicial de água no solo. Após integração de um ano repetiuse o procedimento por 25 vezes até alcançar equilíbrio de água no solo, obtendo-se, assim, o perfil de água nas três camadas. Na pastagem, as frações do conteúdo de água na superfície, $w_{1}$, na zona de raízes, $w_{2}$, e na zona de recarga, $w_{3}$, foram $0,80,0,85$ e $0,90 \mathrm{~m}^{3} \mathrm{~m}^{-3}$, respectivamente, enquanto que na floresta os valores correspondentes foram $0,67,0,70$ e $0,80 \mathrm{~m}^{3} \mathrm{~m}^{-3}$. A temperatura do dossel foi inicializada a partir da temperatura média diária do ar do 


\section{ACTA AMAZONICA}

CALIBRAÇÃO DO "SIMPLIFIED SIMPLE BIOSPHERE MODEL - SSIB"

PARA ÁREAS DE PASTAGEM E FLORESTA NA AMAZÔNIA COM DADOS DO LBA. primeiro dia de medidas, sendo igual a $296 \mathrm{~K}$ para a pastagem e $298 \mathrm{~K}$ para a floresta.

\section{RESULTADOS \& DISCUSSÃO}

Inicialmente, apresenta-se a validação dos fluxos de calor sensível, calor latente, saldo de radiação e fluxo de calor no solo, utilizando-se os parâmetros correspondentes aos dois sítios. Em seguida, mostra-se o resultado da calibração dos parâmetros morfológicos, físicos e fisiológicos para os sítios de pastagem e de floresta, utilizando dados do projeto LBA.

\section{VALIDAÇÃO DO MODELO}

\section{a) Pastagem: Fazenda Nossa Senhora Aparecida - NS}

Os fluxos calculados de saldo de radiação $\left(\mathrm{R}_{\mathrm{n}}\right)$, de calor latente $(\lambda E)$, de calor sensível $(\mathrm{H})$ e de calor no solo $(\mathrm{G})$ versus os observados para o sítio de pastagem são apresentados na Figura 2. Como pode ser visto, o modelo simulou bem o $R_{n}$, fato esse que pode estar relacionado à

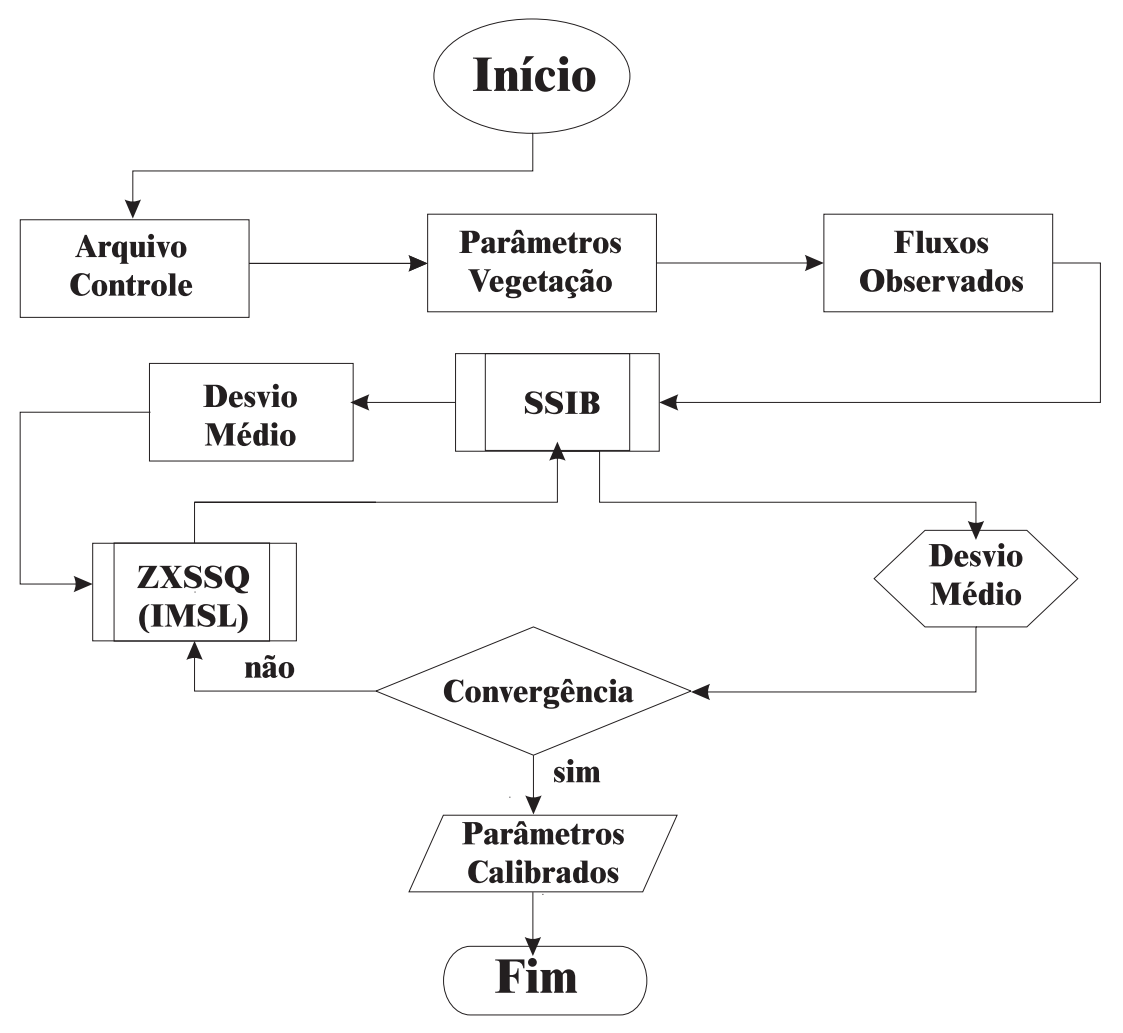

Figura 1 - Diagrama das principais etapas no processo de calibração utilizando o algoritmo de mínimos quadrados ZXSSQ (IMLS, 1984). maneira como ele é calculado no modelo. No modelo SSiB, o $\mathrm{R}_{\mathrm{n}}$ é função da temperatura da superfície e de dois parâmetros morfológicos, a fração de cobertura vegetal $\left(V_{c}\right)$ e o índice de área foliar ( $\mathrm{L})$, conforme mostram as equações A4 e A5 (Apêndice). A boa simulação pode indicar que esses parâmetros representam razoavelmente o sítio de pastagem, pelo menos com respeito ao saldo de radiação. O erro médio ("bias") e o desvio médio quadrático (DMQ) foram pequenos, mostrando mais uma vez que o modelo calculou bem $\mathrm{R}$, conforme pode ser observado na Tabela 1. Nesta tabela, os fluxos correspondem aos valores médios para os períodos de $15 \mathrm{e}$ 14 dias de integração na pastagem e na floresta, respectivamente. Os valores de $\lambda \mathrm{E}$ são superestimados pelo modelo em todo o período, com um valor médio diário de $28,7 \mathrm{~W} \mathrm{~m}^{-2}$ maior que os observados. Isso pode estar relacionado aos parâmetros utilizados no cálculo dessa variável, indicando a necessidade de um melhor ajuste. Os parâmetros responsáveis pelo controle estomático e, conseqüentemente, pela evapotranspiração, são os coeficientes de resistência estomática para a radiação fotossinteticamente ativa $(a, b$ e $c)$, a fração de cobertura vegetal $\left(\mathrm{V}_{c}\right)$ e a fração de folha verde do dossel $\left(\mathrm{N}_{c}\right)$. A diferença entre o fluxo médio de calor sensível modelado e o observado foi menor que aquele para o fluxo de calor latente; contudo, em termos relativos $(\mathrm{DH} / \mathrm{H}$ e $\mathrm{D} \lambda \mathrm{E} / \lambda \mathrm{E})$, o fluxo melhor simulado pelo modelo é o de calor latente, como pode ser visto na Tabela 1. Apesar de apresentar o menor erro médio, o DMQ foi um tanto elevado $\left(39,0 \mathrm{~W} \mathrm{~m}^{-2}\right)$, indicando haver alta dispersão entre os valores observados e os calculados. O fluxo de calor no solo $(G)$ foi razoavelmente calculado pelo modelo. Essa variável foi estimada por resíduo, isto é, através da diferença entre o saldo de radiação e os fluxos de calor latente e calor sensível. Quanto ao armazenamento na biomassa, ele é desprezível.

A Figura 3 apresenta a evolução temporal dos fluxos de superfície, tanto calculados quanto observados. Como já dito, o $\mathrm{R}_{\mathrm{n}}$ foi bem estimado para todos os dias, enquanto $\lambda \mathrm{E}$ foi superestimado durante todo o período. As maiores diferenças ocorrem no intervalo das 10 às $14 \mathrm{HL}$ em praticamente todos os dias. Situação oposta é observada no caso de $\mathrm{H}$, onde o modelo subestimou os valores observados em todos os dias, exceto o dia 17 de setembro (260). Nota-se também alguma defasagem nos fluxos nos horários próximos ao meio dia, o que pode estar relacionado ao método "eddy correlation" utilizado nas 


\section{ACTA \\ AMAZONICA}

CALIBRAÇÃO DO "SIMPLIFIED SIMPLE BIOSPHERE MODEL - SSIB"

PARA ÁREAS DE PASTAGEM E FLORESTA NA AMAZÔNIA COM DADOS DO LBA. medidas destes fluxos, o qual não garante o fechamento do balanço de energia. Como no modelo SSiB o balanço de energia sempre é fechado, isso pode conduzir a diferenças nos resultados. Rocha et al. (1996) encontram resultados semelhantes a esses quando calibraram os parâmetros do modelo SiB (Simple Biosphere Model) utilizando dados coletados durante o projeto ABRACOS. Neste estudo foram utilizados dados de períodos diferentes, com medidas realizadas em dois sítios de pastagem (Fazenda Dimona, AM e Fazenda Nossa Senhora, RO), obtidas com metodologias distintas. Na fazenda Dimona utilizaram-se perfis de psicrômetros e anemômetros montados verticalmente em uma torre de $9 \mathrm{~m}$, calculando-se os fluxos turbulentos com o método da Razão de Bowen, tendo como conseqüência o fechamento do balanço de energia. Como o modelo SiB fecha o balanço de energia, houve uma melhor concordância entre os dados observados e os modelados. Contudo, isso não aconteceu na Fazenda Nossa Senhora, em que as medidas dos fluxos foram realizadas usando o instrumento Hydra ("eddy correlation"), com o qual o fechamento do balanço de energia não é garantido, podendo assim apresentar discrepâncias em comparação com os dados observados. No presente estudo, o balanço de energia também não fecha, uma vez que se utilizou um anemômetro sônico tridimensional, conduzindo assim a possíveis diferenças nos fluxos medidos.

Nos últimos anos tem-se discutido as incertezas e os possíveis erros nas medidas de fluxos usando a técnica de correlação dos vórtices turbulentos sobre terrenos complexos (Finnigan, 1999; Sakai et al., 2001; Finnigan et al., 2003; Kruijt et al. 2003; Von Randow, 2002 e 2004). Von Randow et al. (2004), comparando medidas de fluxos de radiação e de fluxos turbulentos de energia e de $\mathrm{CO}_{2}$ coletadas no período de fevereiro de 1999 a setembro de 2002 no sítio de pastagem Fazenda Nossa Senhora, observaram o não fechamento do balanço de energia, com a soma dos fluxos turbulentos variando de 80 a $110 \%$ da energia disponível. As razões para a aparente subestimativa dos fluxos não são ainda claras, mas, segundo os autores, podem estar relacionadas a dois fatores: primeiro, a lenta mudança na direção do vento sobre terrenos ondulados, adicionando uma componente de baixa freqüência que não pode ser capturada usando rotações de curta escala de tempo no instrumento (Finningan et al. 2003); segundo, a divergência horizontal do vento, que pode causar advecções horizontais dos fluxos de calor sensível e de calor latente, não pode ser estimada através de medidas obtidas numa única torre.

\section{b) Floresta: Reserva Biológica do Jaru - RJ}

Os fluxos calculados de saldo de radiação $\left(R_{n}\right)$, de calor latente $(\lambda \mathrm{E})$, de calor sensível $(\mathrm{H})$ e de calor no solo $(\mathrm{G})$ versus os observados para o sítio de floresta são apresentados na Figura 4. Assim como na pastagem, o modelo calculou bem o R . Esse resultado pode indicar que os parâmetros, utilizados no cálculo do saldo de radiação $\left(\mathrm{V}_{\mathrm{c}}\right.$ e $\left.\mathrm{L}_{\mathrm{t}}\right)$, representam adequadamente $\mathrm{o}$
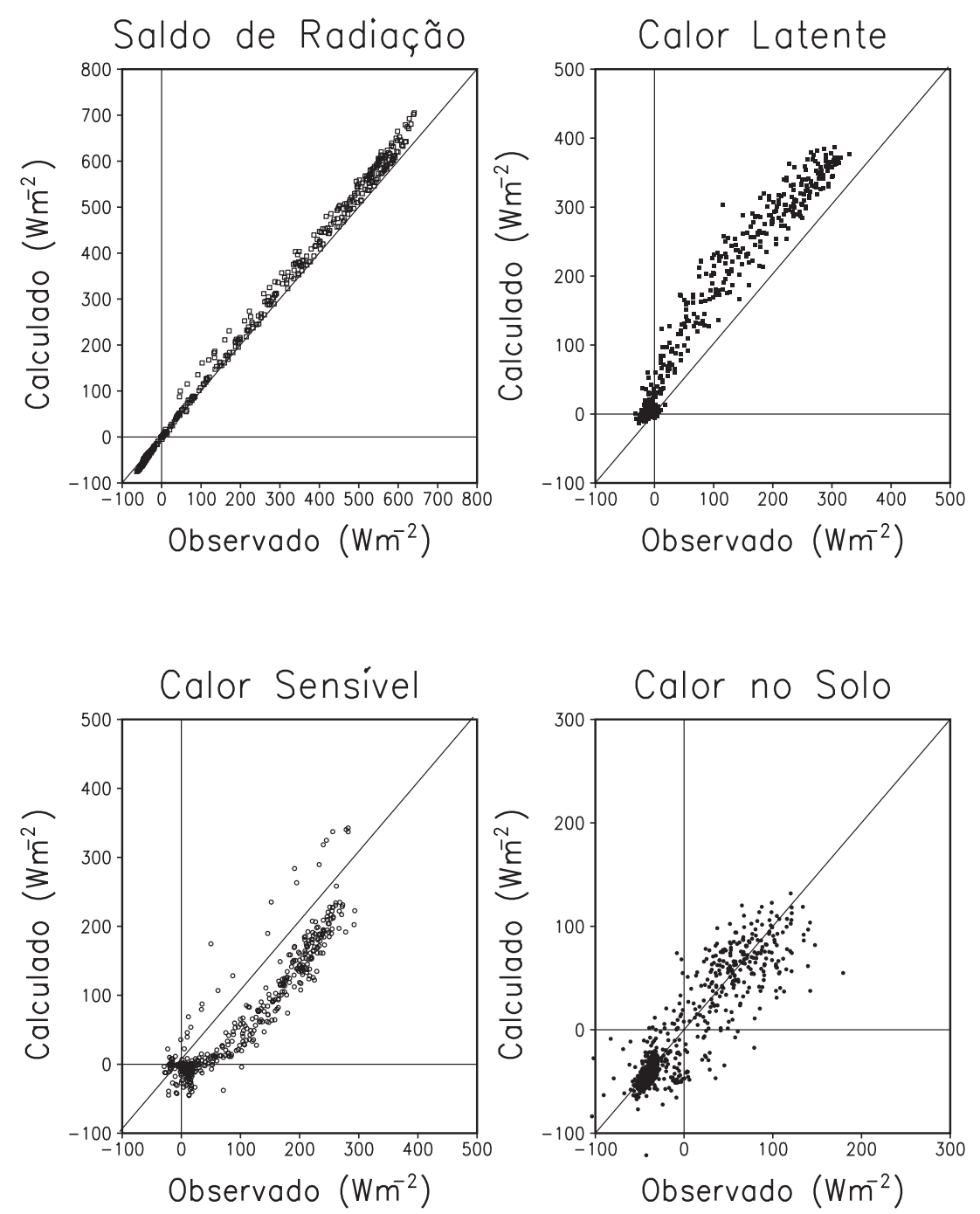

Figura 2 - Fluxos calculados de saldo de radiação $\left(R_{n}\right)$, calor latente $(\lambda E)$ e calor sensível $(\mathrm{H})$ versus os observados no sítio de pastagem Fazenda Nossa Senhora Aparecida (Ano 2001). 


\section{ACTA AMAZONICA}

CALIBRAÇÃO DO "SIMPLIFIED SIMPLE BIOSPHERE MODEL - SSIB"

PARA ÁREAS DE PASTAGEM E FLORESTA NA AMAZÔNIA COM DADOS DO LBA. sítio de floresta. O erro médio foi pequeno $(4,3)$, indicando valores mais próximos aos observados. Concernente aos fluxos de calor latente e calor sensível, uma menor dispersão foi observada no fluxo de calor sensível (ver DMQ na Tabela 1). Assim como na pastagem, o fluxo de calor latente lE é superestimado pelo modelo em todo os dias de simulação, com valor médio em torno de $31 \mathrm{~W} \mathrm{~m}^{-2}$ acima do observado. Esse resultado pode também estar relacionado aos parâmetros utilizados no cálculo dessa variável (coeficientes de resistência estomática para a radiação fotossinteticamente ativa $(a, b$ e $c), \mathrm{V}_{\mathrm{c}} \mathrm{e}$ $\mathrm{N}_{\mathrm{c}}$ ), necessitando de ajuste. O erro médio de $67,0 \mathrm{~W} \mathrm{~m}^{-2}$ confirma que o modelo superestimou o $\lambda$ E. Já $H$ é subestimado, principalmente no período noturno, conforme mostrado na Figura 5 e na Tabela 1. Na média, o H calculado foi da ordem de $10 \mathrm{~W} \mathrm{~m}^{-2}$ menor que o observado, um valor abaixo daquele calculado para a pastagem, o que mostra que, na floresta, Hé mais bem representado pelo modelo. Claramente pode ser visto que o fluxo de calor no solo (G) foi mal simulado, sem nenhuma correlação. Isso é devido ao fato de $\mathrm{G}$ ter sido obtido como resíduo das medidas de saldo de radiação, de calor latente e de calor sensível. A taxa de energia armazenada pela biomassa, no caso da floresta, não foi calculada nesse estudo. Não obstante, Delire e Foley (1999), avaliando o desempenho do modelo "Integrated Biosphere Simulator - IBIS" (Foley et al. 1996) com dados meteorológicos coletados no mesmo sítio de floresta durante o Projeto ABRACOS, obtiveram boa estimativa do saldo de radiação; entretanto, o fluxo de calor no solo foi superestimado, provavelmente pelo modelo não considerar uma camada residual na superfície ou por subestimar o armazenamento de calor no dossel.

Alvaláet al. (2002) analisaram a variação intradiurna e sazonal da temperatura e do fluxo de calor no solo, em áreas de pastagem e de floresta durante o período seco (agosto) e úmido (fevereiro) em Rondônia. Estes autores observaram que o acumulado diário do fluxo de calor no solo na floresta correspondeu a 3\% do saldo de radiação, enquanto que na pastagem esse valor foi cerca de $18 \%$. Os menores valores na floresta são conseqüência da maior quantidade de energia armazenada pela biomassa. Esses resultados mostram que o fluxo de calor no solo não é uma componente desprezível do balanço de energia diário na pastagem, enquanto que na floresta seu valor não é tão significativo. Diferente do fluxo de calor no solo, a taxa de armazenamento de energia na floresta é mais significativa. Diferentes estudos têm demonstrado que a taxa de armazenamento numa camada de vegetação pode ser um componente significativo do balanço de energia quando a quantidade de biomassa é grande (Moore

Tabela 1 - Resultados estatísticos da simulação para pastagem (NS) e floresta (RJ) parâmetros não calibrados (Ano 2001) (W m²).

\begin{tabular}{lcccccccc}
\hline \hline & \multicolumn{3}{c}{ Calculado } & \multicolumn{4}{c}{ Observado } \\
\hline Sítios & Rn & IE & H & G & Rn & IE & H & G \\
\hline NS & 138,1 & 98,9 & 43,6 & $-4,5$ & 126,6 & 70,2 & 61,1 & $-0,1$ \\
RJ & 162,7 & 132,3 & 32,5 & $-1,8$ & 159,4 & 101,2 & 42,5 & 32,0 \\
\hline \multicolumn{4}{c}{ Erro Médio } \\
\hline NS & 7,4 & 38,8 & $-26,9$ & $-8,8$ & 21,4 & 53,6 & 39,0 & 40,8 \\
RJ & 4,3 & 67,0 & $-14,3-44,8$ & 10,8 & 102,1 & 35,4 & 97,9 \\
\hline \hline
\end{tabular}
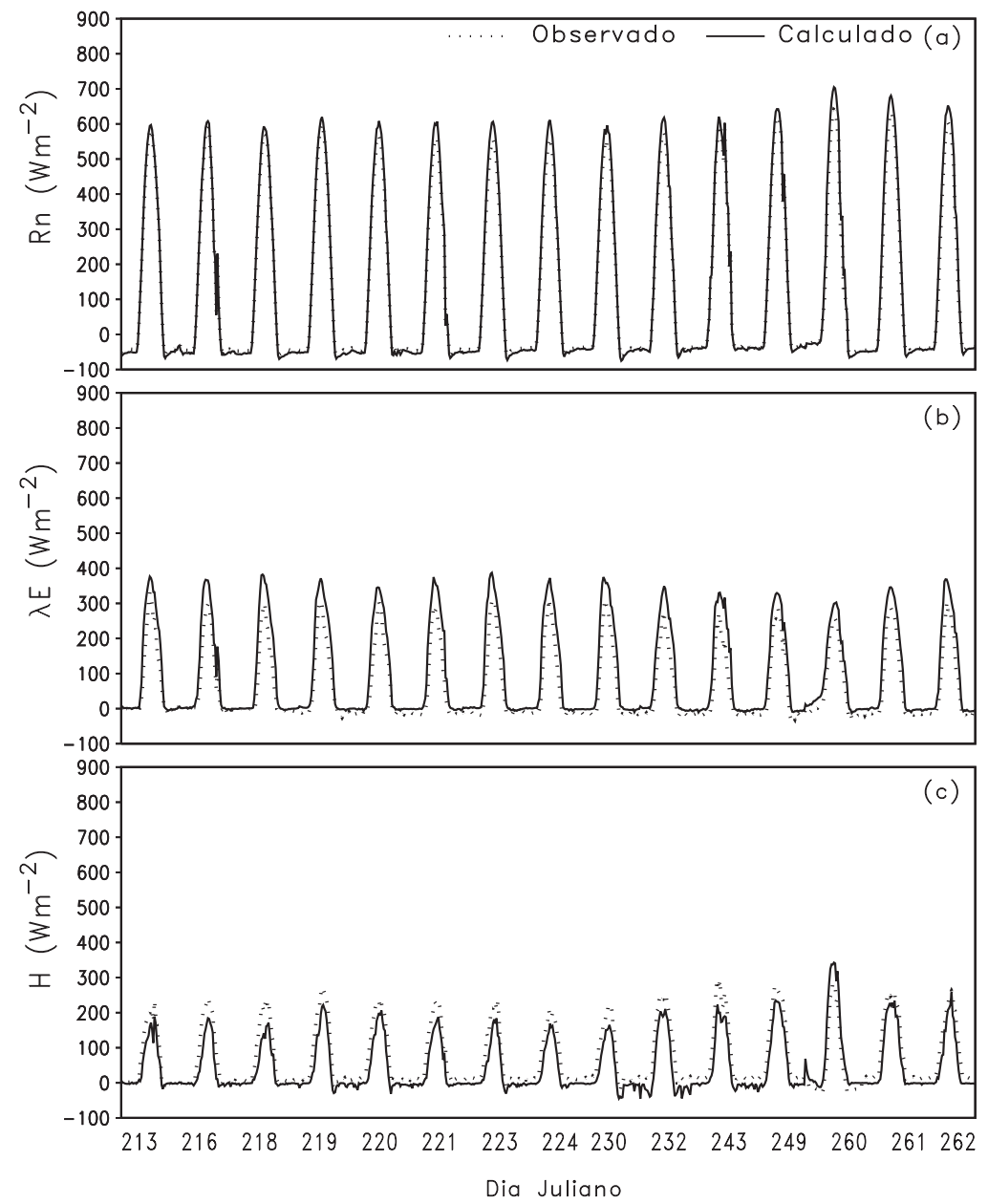

Figura 3 - Evolução temporal dos fluxos de saldo de radiação $\left(R_{n}\right)$, de calor latente (IE) e de calor sensível (H) simulados pelo modelo SSiB (linha sólida) e observados (pontilhados) para o sítio de pastagem Fazenda Nossa Senhora Aparecida (Ano 2002). 


\section{ACTA \\ AMAZONICA}

CALIBRAÇÃO DO "SIMPLIFIED SIMPLE BIOSPHERE MODEL - SSIB"

PARA ÁREAS DE PASTAGEM E FLORESTA NA AMAZÔNIA COM DADOS DO LBA. e Fisch, 1986). Recentemente Michiles (2004), a partir de temperaturas medidas em floresta de Terra Firme, durante a estação seca na Amazônia Central, calculou a taxa de armazenamento de energia nesta floresta, utilizando todos os componentes que armazenam e liberam energia ao longo do dia na floresta: liteira, componentes de pequeno porte (palmáceas, cipós, etc.), troncos, galhos, ramos, folhas e ar. Determinou que um terço da energia é armazenada nos troncos, enquanto outro terço o é no ar; finalmente, o último terço é dividido entre as demais partes. Durante a noite, e em períodos de transição, a energia armazenada na floresta pode ser uma fração bastante significativa, ou mesmo, exceder o saldo de radiação. Em totais diários, observaram-se valores de armazenamento entre 1 e 10\% do saldo de radiação, sendo estes dependentes das condições do tempo ao longo do dia. Diante desses resultados observa-se que o fluxo de calor no solo e a taxa de armazenamento na biomassa desempenham papel importante no balanço de energia à superfície na pastagem e na floresta, respectivamente. Por motivos técnicos, não foi possível medir o fluxo de calor no solo nesse estudo, nem se determinou a taxa de armazenamento na floresta. Não obstante, mesmo sendo o fluxo de calor no solo calculado por resíduo, é importante compará-lo com aquele simulado pelo modelo SSiB.

A Figura 5 apresenta a evolução temporal dos fluxos de superfície tanto calculados quanto observados. Como já mostrado, o modelo calculou bem o $\mathrm{R}_{\mathrm{n}}$ durante todo o período, representando bem o ciclo diurno. As maiores diferenças ocorrem à noite, quando o saldo de radiação é ligeiramente superestimado, o que pode estar relacionado à temperatura da superfície calculada pelo modelo, uma vez que ela é utilizada no cálculo do $\mathrm{R}_{\mathrm{n}}$. $\mathrm{O} \lambda \mathrm{E}$ foi superestimado em todos os dias da simulação. Observam-se também as maiores diferenças no intervalo das 10 às $14 \mathrm{HL}$. O calor sensível apresentou maior diferença durante a noite, em que é subestimado praticamente em todos os dias da simulação. Também se nota no fluxo de calor sensível uma defasagem entre os valores observados e os calculados no ciclo diurno, talvez relacionado ao método utilizado nas medidas de fluxos. Concernente ao fechamento do balanço de energia, Von Randow et al., 2004, ressaltam que os fluxos turbulentos na floresta alcançam aproximadamente $74 \%$ da energia disponível.

\section{CALIBRAÇÃO DOS PARÂMETROS}

As características físicas, morfológicas e fisiológicas da vegetação são usadas para derivar os coeficientes e as resistências que governam as transferências de momentum, de radiação, de fluxos de calor sensível e calor latente (transferência de umidade através do sistema solo-plantaatmosfera). Todos esses fluxos dependem do estado da cobertura vegetal e das condições atmosféricas. Os parâmetros utilizados no cálculo da radiação absorvida são o índice de área foliar $\left(\mathrm{L}_{\mathrm{t}}\right)$, a distribuição do ângulo da folha $\left(\mathrm{X}_{\mathrm{L}}\right)$, a fração de folha verde $\left(\mathrm{N}_{\mathrm{c}}\right)$, a fração de cobertura vegetal $\left(V_{c}\right)$ e as propriedades óticas da folha. O cálculo das resistências estomáticas no modelo SSiB é de vital importância para a estimativa da transferência de vapor d'água da superfície para a atmosfera. Essas resistências são calculadas como o efeito integrado das resistências estomáticas das folhas no dossel das árvores. Os parâmetros fisiológicos importantes usados no cálculo dessas resistências são o índice de área foliar $\left(\mathrm{L}_{\mathrm{t}}\right)$, a fração de folha verde $\left(\mathrm{N}_{\mathrm{c}}\right)$, a fração de
Figura 4 - Fluxos calculados de saldo de radiação $\left(R_{n}\right)$, de calor latente $(\lambda E)$, de calor sensível $(\mathrm{H})$ e de calor no solo $(\mathrm{G})$ versus os observados no sítio de floresta Reserva Biológica do Jaru (Ano 2001).

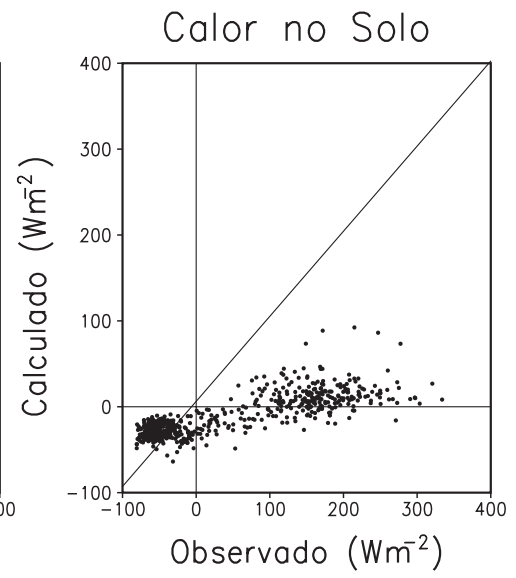


cobertura vegetal $(\mathrm{V})$, os coeficientes de resistência estomática à radiação fotossinteticamente ativa $(a, b$ e $c)$, a porosidade $\left(\mathrm{q}_{\mathrm{s}}\right)$, o parâmetro do potencial de umidade do solo (B) e o parâmetro de resposta estomática à pressão de vapor d'água $\left(h_{5}\right)$. Para a calibração foram utilizados os parâmetros relacionados com a transferência de calor latente e calor sensível entre a superfície e a atmosfera, como mostrado na Tabela 2.

No sítio de pastagem todos os parâmetros iniciais são os de Rocha et al. (1996). O algoritmo necessitou 27 iterações para alcançar a convergência no processo de calibração. O desvio médio do erro (Tabela 2), definido na Equação 2, diminuiu cerca de $70 \%$, isto é, de 4338,9 para 1451,4 . Os erros médios, tanto para $\lambda \mathrm{E}$ quanto para $\mathrm{H}$, diminuíram com os parâmetros calibrados. Estas diminuições ocorrem devido a não-linearidades, apesar de alguns parâmetros para a pastagem mostrarem poucas modificações durante a otimização, e outros, como o comprimento de rugosidade $\left(\mathrm{z}_{0}\right)$ e o deslocamento do plano zero (D), praticamente não se alterarem. A resistência estomática à radiação fotossinteticamente ativa $(a)$ foi o que apresentou maior alteração. A Figura 6 apresenta a evolução temporal dos fluxos de calor latente e calor sensível com os parâmetros calibrados. Pode-se observar na Figura 6c, a qual mostra o ciclo diurno médio, que o erro no fluxo de calor latente diminuiu, aproximando os fluxos calculados pelo modelo com aqueles medidos. Para o fluxo de calor sensível, o erro também diminuiu com os novos parâmetros.

As pequenas alterações nos parâmetros durante a otimização podem estar relacionadas a dois fatores: primeiro, os parâmetros iniciais já estão ajustados para esse sítio, tal que o algoritmo não alcance melhores resultados; segundo, os fluxos de calor latente e calor sensível utilizados na calibração foram obtidos a partir da utilização de anemômetro sônico, através do sistema de correlação de vórtices turbulentos. Embora esse método tenha sido usado satisfatoriamente em vários experimentos na Amazônia e providos dados para outras calibrações (Sellers et al. 1989; Wright et al., 1995; Rocha et al., 1996; Wright et al., 1996), ele não garante o fechamento do balanço de energia, podendo, assim, contribuir para possíveis discrepâncias no procedimento de calibração.

As principais diferenças observadas entre as medidas dos fluxos turbulentos considerados no presente estudo e aquelas reportadas por Sellers et al., 1989; Wright et al., $1996 \mathrm{e}$ Rocha et al., 1996, estão relacionadas com o aperfeiçoamento tanto dos equipamentos de medição, quanto dos algoritmos utilizados nos cálculos dos fluxos. Sellers et al., 1989 utilizaram o instrumento Mark 1 Hydra para medidas de fluxos utilizando a técnica de "eddy correlation". Esse instrumento consistiu de um anemômetro vertical sônico (unidimensional), termômetro termopar, um instrumento de resposta rápida para medida de umidade, e dois anemômetros horizontais montados ortogonalmente, conforme descrito por Shuttleworth et al. (1988). Rocha et al.,1996 utilizaram um instrumento similar, porém com algumas melhorias (ver detalhes em Wright et al., 1995), para realizar a calibração dos parâmetros da Fazenda Nossa Senhora e da Fazenda Dimona. No presente estudo, os instrumentos usados para cálculos dos fluxos turbulentos,
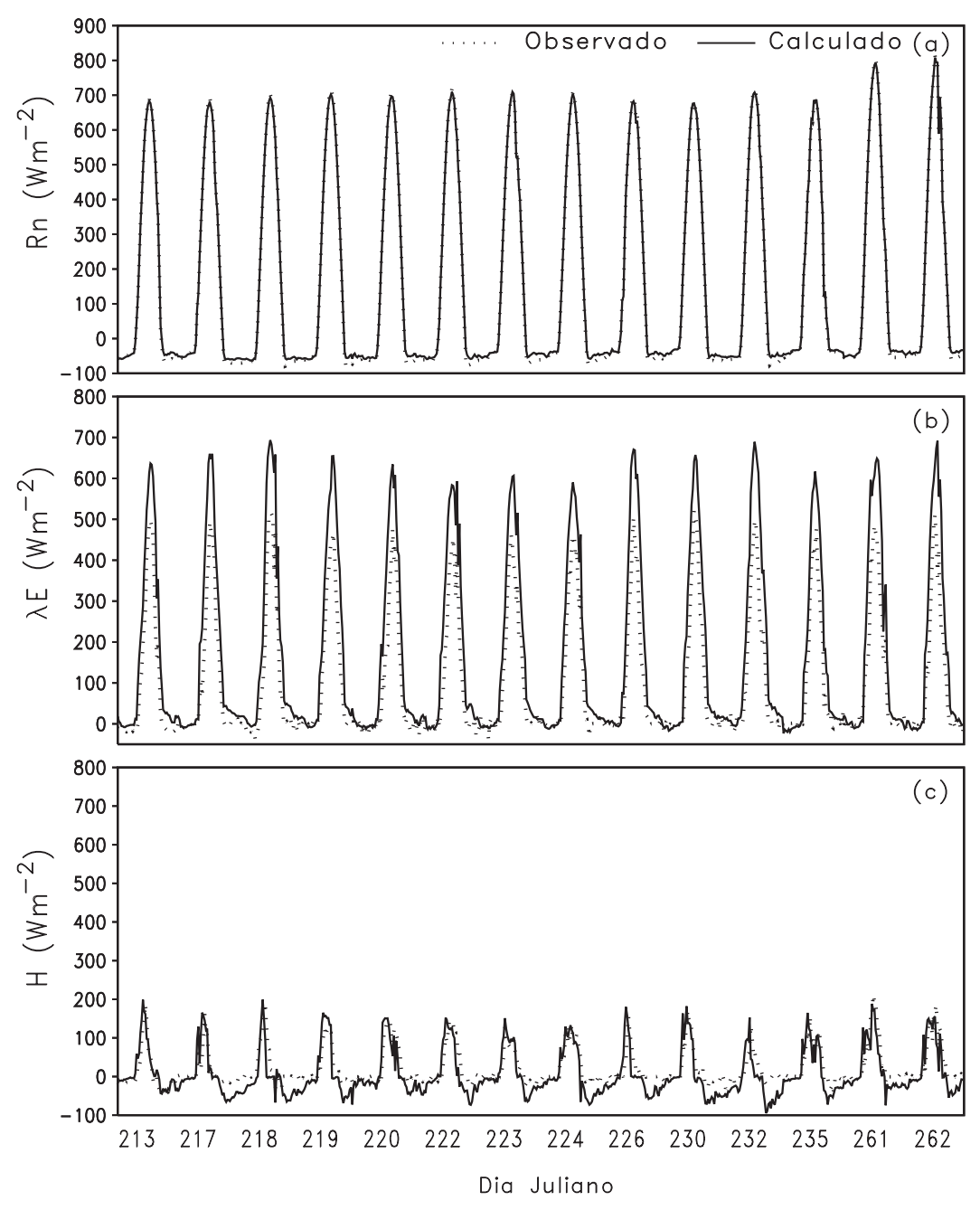

Figura 5 - Evolução temporal dos fluxos de saldo de radiação $\left(R_{n}\right)$, de calor latente (IE) e de calor sensível (H) calculados pelo modelo SSiB (linha sólida) e observados (pontilhados) para o sítio de floresta Reserva Biológica do Jaru (Ano 2001). 


\section{ACTA AMAZONICA}

tanto na floresta quanto na pastagem, são similares àqueles descritos por Moncrieff et al. (1997), os quais são compostos de um anemômetro sônico tridimensional (Solent 1012R2, Gill Instruments, Reino Unido) e um analisador de gás infravermelho de resposta rápida (IRGA, LI-6262, LICOR, Estados Unidos). Para o cálculo dos fluxos utilizou-se o programa Alteddy (Elbers, 1998).

Para o sítio de floresta os parâmetros iniciais utilizados na calibração foram obtidos da literatura. O índice de área foliar $\left(\mathrm{L}_{\mathrm{t}}\right)$, a fração de cobertura vegetal $\left(\mathrm{V}_{\mathrm{c}}\right)$, o deslocamento do plano zero (D), a porosidade $\left(\mathrm{q}_{\mathrm{s}}\right)$ e a condutividade hidráulica do solo à saturação $\left(\mathrm{K}_{\mathrm{s}}\right)$ foram extraídos de Nobre et al. (1991); o parâmetro déficit de pressão de vapor água $\left(\mathrm{h}_{5}\right)$, a fração de folha verde $\left(\mathrm{N}_{\mathrm{c}}\right)$ e os potenciais de água no solo $\left(\mathrm{y}_{1}\right.$ e $\left.\mathrm{y}_{2}\right)$ foram extraídos de Sellers et al. (1989); enquanto os coeficientes de resistência estomática à radiação fotossinteticamente ativa $(a, b$ e $c)$ foram extraídos de Dorman \& Sellers (1989). O algoritmo alcançou a convergência após 27 iterações. Resultados semelhantes àqueles encontrados para a pastagem foram obtidos para o sítio de floresta (Tabela 2). Alguns parâmetros apresentaram poucas mudanças na otimização. Embora o desvio médio do erro tenha diminuído menos do que na pastagem, os erros médios dos fluxos de calor latente e calor sensível praticamente diminuíram na mesma ordem que para a pastagem. A Figura 7 apresenta a evolução temporal dos fluxos de calor latente e calor sensível com os parâmetros calibrados.

Pode-se observar que o erro para o fluxo de calor latente diminuiu em relação aos parâmetros não calibrados, aproximando os fluxos calculados daqueles observados. Para o fluxo de calor sensível pouca variação foi observada; porém, diminuição no erro foi observada (Tabela 2). Esses resultados mostram que, com os parâmetros calibrados, o modelo simulou satisfatoriamente os fluxos de superfície. Assim como na pastagem, pequenas mudanças nos parâmetros podem estar relacionadas aos fatores acima mencionados.

A Tabela 3 apresenta os componentes do balanço de energia após o procedimento de calibração dos parâmetros para os sítios de pastagem e de floresta. Os fluxos de calor latente e de calor sensível apresentaram redução (12\%) e aumento (21\%), respectivamente, diminuindo o desvio com relação aos fluxos observados, como também pode ser visto na Tabela 3. O saldo de radiação apresentou ligeira redução, enquanto que a mudança no fluxo de calor no solo não foi muito significativa. Esses resultados mostram mais uma vez que os parâmetros ajustados são mais apropriados para representar os processos de transferência turbulenta entre a superfície e a atmosfera para ambos os sítios.

Uma questão que se levanta é se os parâmetros que foram calibrados considerando um período curto de dados

Tabela 2 - Resultados do processo de otimização.

\begin{tabular}{|c|c|c|c|c|}
\hline \multirow[b]{2}{*}{ Parâmetros } & \multicolumn{2}{|c|}{ Pastagem (NS) } & \multicolumn{2}{|c|}{ Floresta (RJ) } \\
\hline & Inicial & Calibrado & Inicial & Calibrado \\
\hline Índice de área foliar - Lt $\left(\mathrm{m}^{2} \mathrm{~m}^{-2}\right)$ & 1,610 & 1,530 & 5,000 & 4,819 \\
\hline Fração de folha verde $-N_{c}$ & 0,930 & 0,901 & 0,950 & 0,912 \\
\hline Fração de cobertura vegetal $-V_{c}$ & 0,790 & 0,742 & 1,000 & 0,927 \\
\hline \multicolumn{5}{|c|}{ Parâmetros de resistência estomática à radiação PAR } \\
\hline$\left(\mathrm{J} \mathrm{m}^{-3}\right)$ & 11554,0 & 11591,4 & 2336,0 & 2357,1 \\
\hline$\left(\mathrm{W} \mathrm{m}^{-2}\right)$ & 2,100 & 2,899 & 0,010 & 0,093 \\
\hline$\left(\mathrm{s} \mathrm{m}^{-1}\right)$ & 110,00 & 107,90 & 154,00 & 150,89 \\
\hline Comprimento de rugosidade $-\mathrm{z}_{\mathrm{o}}(\mathrm{m})$ & 0,022 & 0,020 & 2,650 & 2,550 \\
\hline Deslocamento do plano zero - D (m) & 0,170 & 0,200 & 27,400 & 29,102 \\
\hline Porosidade $-\ominus_{\mathrm{s}}\left(\mathrm{m}^{3} \mathrm{~m}^{-3}\right)$ & 0,460 & 0,490 & 0,420 & 0,482 \\
\hline $\begin{array}{l}\text { Condutividade hidráulica à saturação - Ks } \\
\left(\mathrm{m} \mathrm{s}^{-1}\right)\end{array}$ & $1,0 \times 10-5$ & $1,5 \times 10-5$ & $2,0 \times 10-5$ & $4,6 \times 10-6$ \\
\hline $\begin{array}{l}\text { Parâmetro do déficit de pressão de vapor } \\
\text { d'água - h5 (hPa-1) }\end{array}$ & 0,0184 & 0,0165 & 0,0222 & 0,0201 \\
\hline \multicolumn{5}{|l|}{ Parâmetros do potencial de água no solo } \\
\hline$\psi 1(\mathrm{~m})$ & 1,800 & 1,850 & 1,200 & 1,190 \\
\hline$\psi 2(\mathrm{~m})$ & 5,670 & 5,777 & 6,250 & 6,270 \\
\hline Desvio médio do erro - ( $\mathrm{W} \mathrm{m}-2)$ & 4338,9 & 1451,4 & 240,9 & 142,7 \\
\hline Erro médio ("bias") de $\lambda E \quad(\mathrm{~W} \mathrm{~m}-2)$ & 98,9 & 86,3 & 132,3 & 117,4 \\
\hline Erro médio ("bias") de $\mathrm{H}$ (W m-2) & 43,6 & 55,6 & 32,5 & 38,5 \\
\hline
\end{tabular}


(15 dias da estação seca de 2001) apresentam o mesmo desempenho (melhora na estimativa dos fluxos turbulentos) se forem utilizados na simulação para outro período. Logo, o modelo SSiB foi integrado para o mesmo período no ano seguinte (2002). As Figuras 8 e 9 apresentam, respectivamente, a evolução temporal dos fluxos de calor latente e de calor sensível com os

Tabela 3 - Resultados estatísticos da simulação para pastagem (NS) e floresta (RJ) após calibração dos parâmetros (Ano 2001) (W m²).

\begin{tabular}{|c|c|c|c|c|c|c|c|}
\hline \multirow[b]{2}{*}{ Sítios } & \multicolumn{3}{|c|}{ Calculado } & \multicolumn{4}{|c|}{ Observado } \\
\hline & $\mathrm{Rn}$ & $\lambda E$ & H G & $\mathrm{Rn}$ & $\lambda E$ & $\mathrm{H}$ & G \\
\hline NS & 132,4 & 86,3 & $55,6-3,5$ & 126,6 & 70,2 & 61,1 & $-0,1$ \\
\hline \multirow[t]{2}{*}{ RJ } & 160,3 & 117,4 & $38,5-2,0$ & 159,4 & 101,2 & 42,5 & 32,0 \\
\hline & \multicolumn{3}{|c|}{ Erro Médio } & \multicolumn{4}{|c|}{ DMQ } \\
\hline NS & 5,2 & 30,8 & $-19,2-7,8$ & 21,4 & 51,2 & 30,5 & 39,1 \\
\hline RJ & 3,1 & 60,4 & $-11,3-40,1$ & 8,2 & 100,1 & 31,2 & 94,2 \\
\hline
\end{tabular}
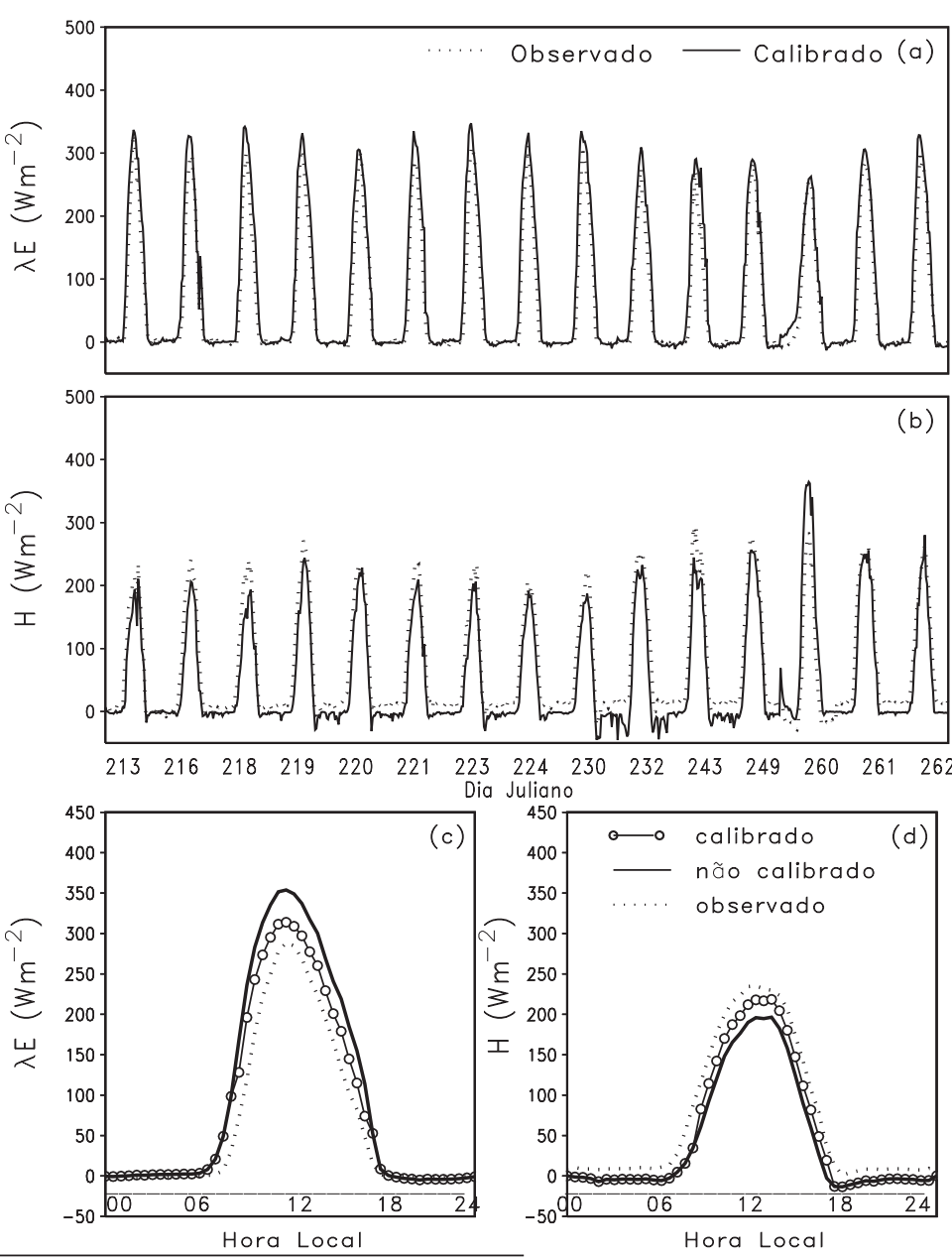

Figura 6 - Fluxos calibrados de calor latente (a) e de calor sensível (b) versus os observados no sítio de pastagem Fazenda Nossa Senhora Aparecida (NS). Ciclos diurnos médios dos fluxos de calor latente (c) e de calor sensível (d) (Ano 2001). parâmetros calibrados no ano de 2002 para os sítios de pastagem e de floresta. Pode-se observar nas figuras, as quais mostram os ciclos diurnos médios, que o erro no fluxo de calor latente também diminui, tanto na floresta nastagem, mostrando assim que existe uma redução na diferença entre os fluxos calculados e os observados, após o ajuste dos parâmetros. Para o fluxo de calor sensível, o erro também diminuiu com os novos parâmetros.

As Tabelas 4 e 5 apresentam, respectivamente, os componentes do balanço de energia para pastagem e para floresta, utilizando os parâmetros não calibrados e calibrados referentes ao ano de 2002. Pode-se observar que os desvios entre os fluxos turbulentos de calor latente e calor sensível calculados e os observados para 2002 também apresentam reduções. Para o fluxo de calor latente, a redução no desvio foi em torno de $10 \mathrm{~W} \mathrm{~m}^{-2}$ para a pastagem e de $13,2 \mathrm{~W} \mathrm{~m}^{-2}$ para o sítio de floresta. Reduções da mesma ordem também foram observadas para o fluxo de calor sensível. O saldo de radiação e o fluxo de calor no solo, como em 2001, também não apresentaram mudanças significativas. Isso de certa forma é esperado, pois os parâmetros que foram utilizados na calibração são usados diretamente no cálculo dos fluxos turbulentos de calor latente e de calor sensível. A redução entre os fluxos calculados e os observados pode ser verificada também no cálculo do erro médio, que apresenta reduções da ordem de $12,8 \mathrm{~W} \mathrm{~m}^{-2}$ para a pastagem e de $9,2 \mathrm{~W} \mathrm{~m}^{-2}$ para a floresta.

\section{CONCLUSÕES}

Considerando que o modelo $\mathrm{SSiB}$ necessita muitos parâmetros de entrada e que alguns destes podem apresentar incertezas quanto às suas especificações ou não ter nenhuma medida de campo associada aos mesmos, faz-se necessário que eles sejam ajustados ou calibrados, visando melhor representar as características físicas e fisiológicas da superfície. Neste trabalho, utilizaram-se medidas micrometeorológicas coletadas na floresta da Reserva Biológica do Jaru e na área de pastagem da Fazenda Nossa 
Tabela 4 - Resultados estatísticos da simulação para pastagem (NS) e floresta (RJ) parâmetros não calibrados (Ano 2002) ( $\left.\mathrm{W} \mathrm{m}^{-2}\right)$.

\begin{tabular}{lcccccccc}
\hline \hline & \multicolumn{3}{c}{ Calculado } & \multicolumn{4}{c}{ Observado } \\
\hline Sítios & Rn & $\lambda \mathbf{E}$ & H & G & Rn & $\lambda \mathbf{E}$ & H & G \\
\hline NS & 149,4 & 107,0 & 40,1 & $-2,3$ & 130,6 & 73,4 & 63,0 & $-5,8$ \\
RJ & 163,7 & 113,6 & 47,5 & $-2,6$ & 155,4 & 79,2 & 57,2 & 19,0 \\
\hline \multicolumn{4}{c}{ Erro Médio } \\
\hline NS & 9,8 & 42,8 & $-22,7$ & $-10,8$ & 31,2 & 64,6 & 45,5 & 31,1 \\
RJ & 4,6 & 50,3 & $-35,3-37,2$ & 18,5 & 98,2 & 23,4 & 84,7 \\
\hline \hline
\end{tabular}

Tabela 5 - Resultados estatísticos da simulação para pastagem (NS) e floresta (RJ) parâmetros calibrados (Ano 2002) (W m²).

\begin{tabular}{|c|c|c|c|c|c|c|c|c|}
\hline \multirow[b]{2}{*}{ Sítios } & \multicolumn{4}{|c|}{ Calculado } & \multicolumn{4}{|c|}{ Observado } \\
\hline & $\mathrm{Rn}$ & $\lambda E$ & $\mathrm{H}$ & G & $\mathrm{Rn}$ & $\lambda E$ & $\mathrm{H}$ & G \\
\hline NS & 146,5 & 97,0 & 48,2 & $-1,3$ & 130,6 & 73,4 & 63,0 & $-5,8$ \\
\hline \multirow[t]{2}{*}{ RJ } & 160,4 & 100,4 & 58,1 & $-1,9$ & 155,4 & 79,2 & 57,2 & 19,0 \\
\hline & \multicolumn{4}{|c|}{ Erro Médio } & \multicolumn{4}{|c|}{ DMQ } \\
\hline NS & 8,3 & 30,3 & $-11,7$ & $-9,2$ & 29,2 & 55,6 & 35,6 & 25,1 \\
\hline RJ & 3,8 & 41,1 & $-22,5$ & $-30,9$ & 17,8 & 96,4 & 13,7 & 76,7 \\
\hline
\end{tabular}
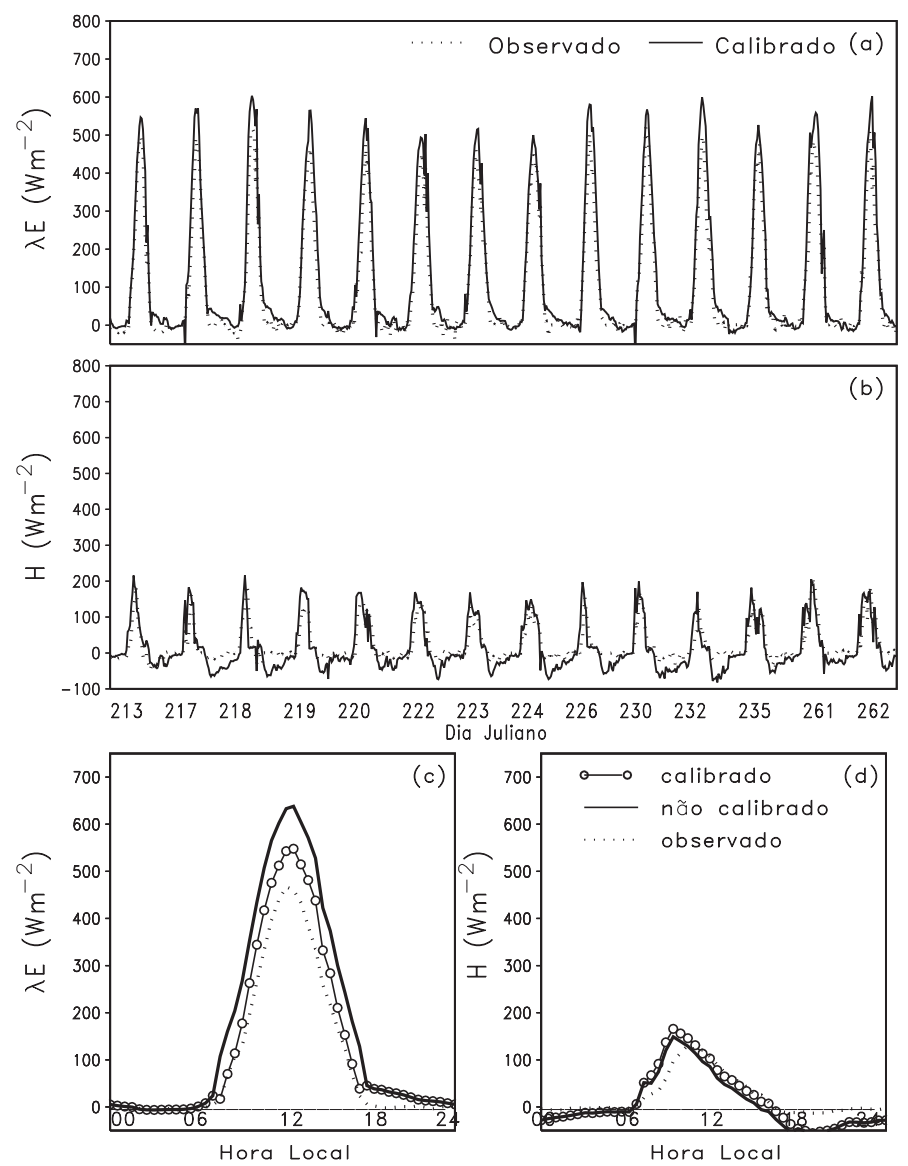

Figura 7 - Fluxos calibrados de calor latente (a) e de calor sensível (b) versus os observados no sítio de floresta da Reserva Biológica do Jaru (RJ). Ciclos diurnos médios dos fluxos de calor latente (c) e de calor sensível (d) (Ano 2001).
Senhora Aparecida, ambas em Rondônia, para validar e calibrar o modelo SSiB numa versão "off-line". No procedimento de calibração utilizou-se o algoritmo de minimização de mínimos quadrados ZXSSQ (IMSL, 1984).

Com os parâmetros não calibrados o modelo simulou bem o saldo de radiação $\left(\mathrm{R}_{\mathrm{n}}\right)$, tanto na pastagem quanto na floresta. $\mathrm{O}$ fluxo de calor latente $(\lambda E)$ foi superestimado nos dois sítios para todos os períodos de simulação, o que deve estar relacionado aos parâmetros utilizados no cálculo dessa variável (os coeficientes de resistência estomática para a radiação fotossinteticamente ativa, a fração de cobertura vegetal e a fração de folha verde), os quais necessitam, portanto, de ajustes. $\mathrm{O}$ modelo subestimou o fluxo de calor sensível $(\mathrm{H})$ na pastagem e na floresta, principalmente no período noturno; porém, para a floresta os valores foram mais próximos daqueles observados. Como o valor do fluxo de calor no solo (G) em ambos os sítios foi obtido como resíduo do balanço de energia, resíduo este que nono caso da floresta inclui a taxa de armazenamento na biomassa, não se conseguiu estimá-lo satisfatoriamente utilizando o modelo.

$\mathrm{O}$ novo conjunto de parâmetros ajustados do modelo SSiB produziu melhores resultados na determinação dos fluxos de calor latente e de calor sensível e, conseqüentemente, representou melhor a partição de energia na floresta e na pastagem. Isso pode ser observado na diminuição do desvio médio quadrático e nos erros médios destes fluxos, apesar de alguns parâmetros, tais como o comprimento de rugosidade e o deslocamento do plano zero na pastagem, terem-se modificado pouco na otimização. Isso deve estar relacionado aos próprios parâmetros, possivelmente já ajustados para o sítio. Uma simulação adicional foi realizada considerando o mesmo período do ano de 2002, com o propósito de verificar se os parâmetros calibrados com dados do ano anterior também apresentavam resultados satisfatórios. Observou-se que os desvios entre os fluxos 


\section{ACTA AMAZONICA}
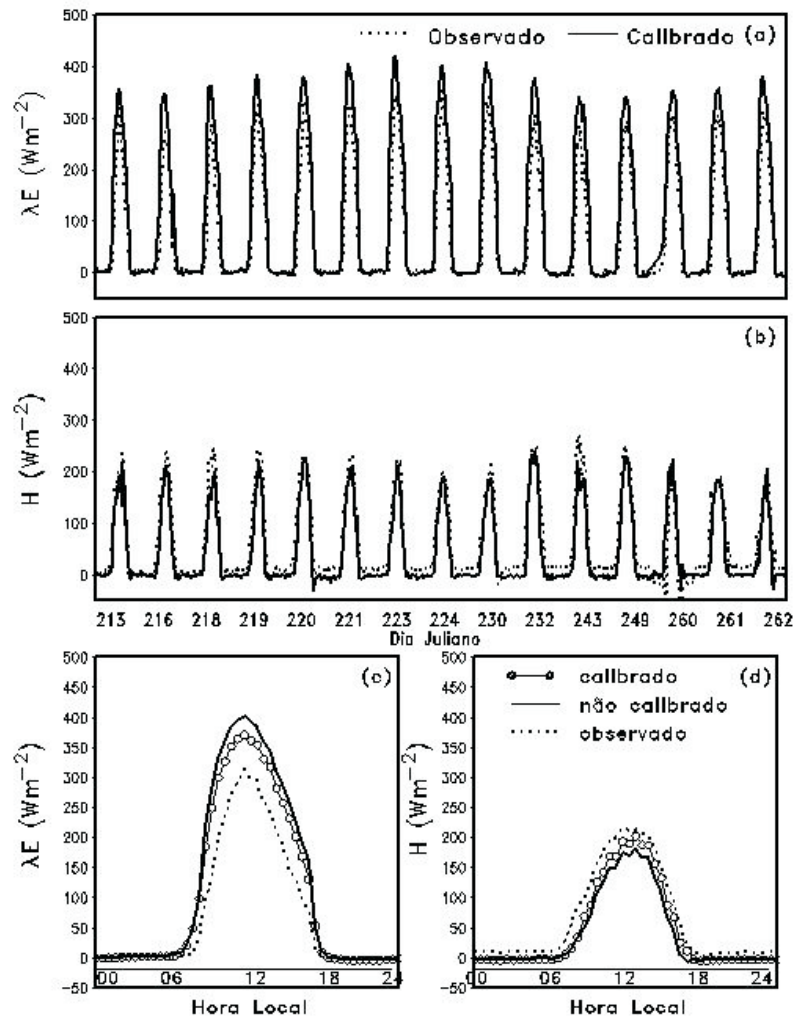

Figura 8 - Fluxos calibrados de calor latente (a) e de calor sensível (b) versus os observados no sítio de floresta da FNS. Ciclos diurnos médios dos fluxos de calor latente (c) e de calor sensível (d) (Ano 2002).

observados e os calculados (latente e sensível) apresentaram reduções, enquanto que mudanças no saldo de radiação e no fluxo calor do solo não foram tão significativas. Isso deve estar relacionado ao fato de os parâmetros calibrados estarem diretamente ligados ao cálculo dos fluxos turbulentos fazendo com que os mesmos apresentem variações mais significativas.

Diversos parâmetros de entrada no modelo SSiB apresentam variação sazonal (fração de folha verde, índice de área foliar, comprimento de rugosidade, deslocamento do plano zero) e, considerando que neste trabalho utilizou-se somente um período do ano (agosto e setembro) para realização da calibração, faz-se necessário ajustar os parâmetros sazonais para diferentes períodos do ano, a fim de melhor representar suas variações.

\section{AGRADECIMENTOS}

Os autores agradecem ao Dr. Humberto Ribeiro da Rocha pelo auxílio na discussão dos resultados. O primeiro autor agradece à Coordenação de Aperfeiçoamento de Pessoal de Nível Superior-CAPES pela concessão de bolsa de doutorado.
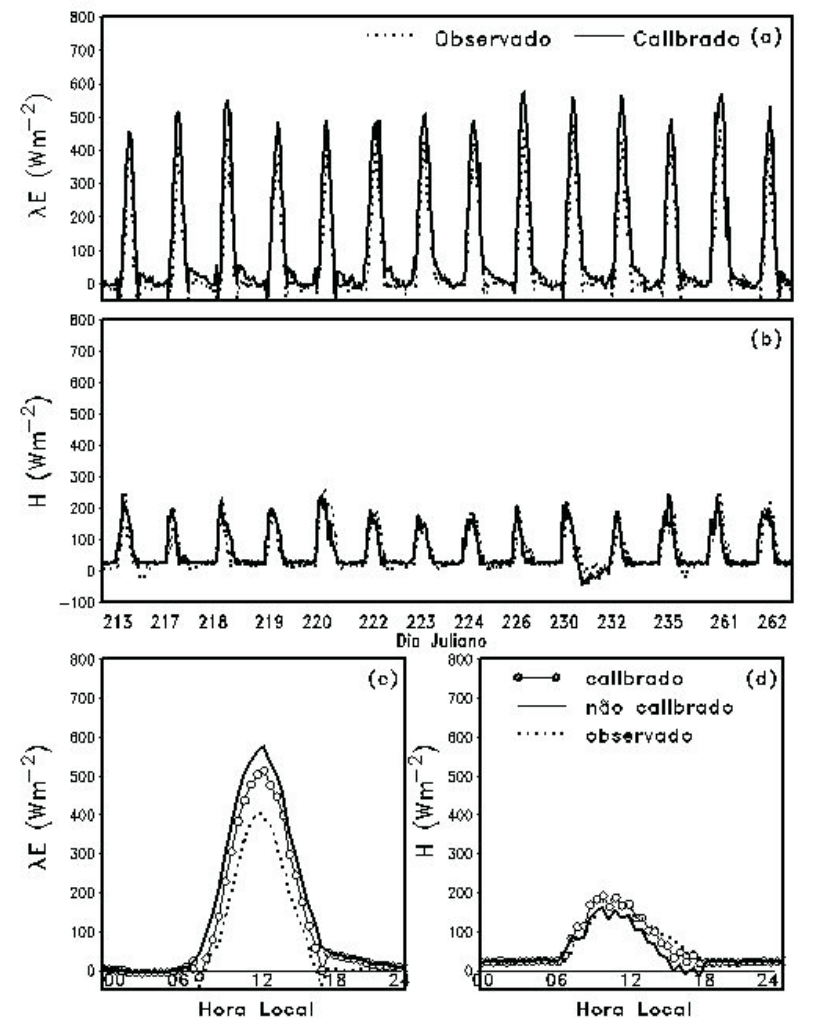

Figura 9 - Fluxos calibrados de calor latente (a) e de calor sensível (b) versus os observados no sítio de floresta da RBJ. Ciclos diurnos médios dos fluxos de calor latente (c) e de calor sensível (d) (Ano 2002).

\section{BIBLIOGRAFIA CITADA}

Alvalá, R. C.; Gielow, R.; Rocha, R.; Freitas, C.; Lopes, M.; Manzi, A.; Von Randow, C.; Dias, M. A. F.; Cabral, O. 2002. Intradiurnal and seasonal variability of soil temperature, heat flux, soil moisture content, and thermal properties under Forest and pasture in Rondônia. Journal of Geophysical Research, 107: no. D20, 10.1029/2001JD000599, 18-1 - 18-20.

Anglo-Brazilian Amazonian Climate Observation Study ABRACOS. Amazonian Deforestation and Climate [on line], 1996. http://www.cptec.inpe.br/abracos. Abril 2002.

Bonan, G.B.; Pollard, D.; Thompson, S.L. 1992. Effects of boreal forest vegetation on global climate. Nature, 359: 716-718.

Charney, J.G. A. 1975. Dinamics of deserts and drought in Sahel. Quarterly Journal of the Royal Meteorological Society, 114: 439-462.

Costa, M.H.; Foley, J.A. 2000. Combined effects of desforestation and doubled atmospheric $\mathrm{CO}_{2}$ concentration on the climate of Amazonia. Journal of Climate,13: 18-34.

Culf, A.D.; Fisch, G.; Hodnett, M.G. 1995. The albedo of Amazonian forest and ranch land. Journal of Climate, 8: 1544-1554. 
Delire, C.; Foley, J.A. 1999. Evaluating the performance of a land surface/ ecosystem model with biophysical measurements from contrasting environments. Journal of Geophysical Research, 104: 16,895-16,909.

Dickinson, R.E.; Kennedy, P.J.; Wilson, M.F. 1986. Biosphereatmosphere transfer scheme (BATS) for the NCAR community climate model. NCAR/TN - $275+$ STR.

Dirmeyer, P.A.; Zeng, F.J.; Ducharne, A.; Morrill, J.C.; Koster, R.D. 2000. The sensitivity of surface fluxes to soil water content in three land surface schemes. Journal of Hydrometeorology, 1: 121-134.

Dorman, J.L.; Sellers, P.J. 1989. A global climatology of albedo, roughness length and stomatal resistance for atmospheric general circulation models as represented by the Simple Biosphere Model (SiB). Journal of Applied Meteorology, 28: 833-855.

Dolman, J.; Gash, J.; Roberts, J.; Shuttleworth, J. 1991. Stomatal and surface conductance of tropical rainforest. Agricultural and Forest Meteorology, 54: 303-318.

Elbers, J. 1998. Eddy correlation system Winand Staring Centre, user manual version 2.0 internal report. Alterra, Wageningen, The Netherlands, 39pp.

Experimento de Grande Escala da Biosfera-Atmosfera na Amazônia - LBA [on line], 1998. http://ba.cptec.inpe.br/ lba/. Fevereiro 2003.

Flerchinger, G.N.; Kustas, W.P.; Weltz, M.A. 1998. Simulating surface energy fluxes and radiometric surface temperatures for two arid vegetation communities using the SHAW model. Journal of Applied Meteorology, 37(5): 449-460.

Finnigan, J. A comment on the paper by Lee (1998) On micrometeorological observation of surface-air exchange over tall vegetation. 1999. Agricultural and Forest Meteorological, 97: 55-64.

Finnigan, J. Clement, R.; Malhi, Y.; Leuning, R.; Cleugh, H. 2003. A re-evaluation of long-term flux measurement techniques. Part I: Averaging and coordinate rotation. Boundary Layer Meteorology. 107: 1-48.

Foley, J.A.; Kutzbach, J.E.; Coe, M.T.; Levis, S. 1994. Feedbacks between climate and boreal forests during the Holocene epoch. Nature, 317: 52-54.

Foley, J.A.; Prentice, C.I.; Ramankutty, N.; Levis, S.; Pollard, D.; Sitch, S.; Haxeltine. 1996. An integrated biosphere model of land surface processes, terrestrial carbon balance, and vegetation dynamics. Global Biogeochemical Cycles, 10: 603-628.

Gash, J.C.; Nobre, C.; Roberts, J.; Victoria, R. 1996. An overview of ABRACOS. In: Gash, J.C.; Nobre, C.; Roberts, J.; Victoria, R. (Eds). Amazonian Deforestation and Climate, John Wiley, New York, p. 1-14.

IMSL (International Mathematical and Statistical Library), 1984. Chapter Z. IMSL, ZXSSQ-1 to ZXSSQ-7.

Kruijt, B.; Elbers, J.; von Randow, C.; Araujo, A.; Culf, A; Bink, N.; Oliveira, P.; Manzi, A.; Nobre, A.; Kabat, P.; Moors, E. The robustness in eddy correlation fluxes for Amazon rainforest conditions . LBA Special issue Ecological Applications (in press).
Manzi, A.O.; Planton, S. 1994. Implementation of the ISBA parametrization scheme for land surface processes in a GCM - an annual cycle experiment. Journal of Hydrology, 155: 353-387.

Manzi, A.O.; Planton, S. 1996. Calibration of a GCM using ABRACOS and ARME data and simulation of Amazonian deforestation. In: Gash, J.C.; Nobre, C.; Roberts, J.; Victoria, R. (Eds). Amazonian Deforestation and Climate, John Wiley, New York, p. 505-529.

McWilliam, A.L. Cabral, O.M.R.; Gomes, B.M.; Esteves, J.L.; Roberts, J.M. 1996. Forest and pasture leaf-gas exchange in south-west Amazonia. In: Gash, J.C.; Nobre, C.; Roberts, J.; Victoria, R. (Eds). Amazonian Deforestation and Climate, John Wiley, New York, p. 265-285.

Michiles, A. A. 2004. Taxas de armazenamento de energia e fluxos de calor nos troncos em floresta de terra firme na Amazônia Central. Dissertação de Mestrado em Meteorologia, INPE, São José dos Campos, SP.

Moore, C.; Fisch, G. 1986. Estimating heat storage in Amazonian tropical forests. Agricultural and Forest Meteorology. 38: 147-169.

Moncrieff, J.; Massheder, J.; De Bruin, H.; Elbers, J.; Friborg, T.; Heusinkveld, B.; Kabat, P.; Scott, S.; Soegaard, H.; Verhoef, A. 1997. A system to measure surface fluxes of momentum, sensible heat, water vapor and carbon dioxide. Journal of Hydrometeorology, 188-189: 589-611.

Nagai, H. 2002. Validation and sensitivity analysis of a new atmosphere-soil-vegetation model. Journal of Applied Meteorology, 41: 160-176.

Nagai, H. 2003. Validation and sensitivity analysis of a new atmosphere-soil-vegetation model. Part II: Impacts on incanopy latent heat flux over a winter wheat field determined by detailed calculation of canopy radiation transmission and stomatal resistence. Journal of Applied Meteorology, 42(3): 434-451.

Nobre, C. A.; Sellers, P. J.; Shukla, J. 1991. Amazonian Deforestation and Regional Climate Change. Journal of Climate, 4: 957-987.

Rocha, H. R; Nobre, C. A.; Bonatti, J. P.; Wright, I. R.; Sellers, P. J. 1996. A vegetation atmosphere interaction study for Amazonian deforestation using field data and a 'single column model. Quarterly Journal Royal Meteorological Society, 122: 567-594.

Sato, Y. C.; Sellers, P. J.; Randall, D. A.; Schneider, E. K.; Shukla, J. Kinter III, J. L.; Hou, Y. T.; Albertazzi, E. 1989. Effects of implementing the Simple Biosphere model in a General Circulation Model. Journal of the Atmospheric Sciences, 46(18): 2757-2782.

Sakai, R.; Fitzjarald, D. Moore, K. 2001. Importance of lowfrequency contributions to eddy fluxes observed over rough surfaces. Journal of Applied Meteorology, 40: 2178-2192.

Sellers, P.J. 1985. Canopy reflectance, photosynthesis and transpiration. International Journal of Remote Sensing, 6: 1335-1372. 
Sellers, P. J.; Mintz, Y.; Sud, Y.C.; Dalcher, A. 1986. A Simple Biosphere Model $(\mathrm{SiB})$ for use within general circulation models. Journal of Atmospheric Sciences, 43: 505-531.

Sellers, P.J.; Dorman, J.L. 1987. Testing the Simple Biosphere Model ( $\mathrm{SiB}$ ) using point micrometeorological and biophysical data. Journal of Applied Meteorology, 26: 622-651.

Sellers, P. J.; Shuttleworth, W. J.; Dorman J. 1989. Calibrating the Simple Biosphere Model for Amazonian Tropical Forest using field and remote sensing data. Part I: Average calibration with field data. Journal of Applied Meteorology, 28: 727-759.

Shuttleworth, W.; Gash, J.; Lloyd, C.; McNeil, D. Moore, C.; Wallace, J. 1988. An integrated micrometeorological system for evaporation measurement. Agricultural and Forest Meteorology, 43: 295-317.

Von Randow, C.; Sá, L.; Gannabathula, P. S.; Manzi, A.; Arlino P.; Kruijt, B. 2002. Scale variability of atmospheric surface layer fluxes of energy and carbon over a tropical rain forest in southwest Amazonia. I. Diurnal conditions. Journal of Geophysical Research, 107, 8062, doi: 10.1029/2001JD000379.

Von Randow, C.; Manzi, O.; Kruij, B.; Oliveira, P.; Zanchi, F.; Silva, R.; Hodnett, M.; Gash, J.; Elbers, J.; Waterloo, M.; Cardoso, F.; Kabat, P. 2004. Comparative measurements and seasonal variations in energy and carbon exchange over forest and pasture in South West Amazonia. Theoretical and Applied Climatology, 78: 5-26.
Wright, I. R.; Manzi, A. O.; Rocha, H. R. Surface conductance of Amazonian pasture: model application and calibration for canopy climate. Agricultural and Forest Meteorology. ,v. 75: p. 51-70, 1995.

Wright, I. R.; Gash, J.; Rocha, H.; Roberts, J. 1996. Modelling surface conductance for Amazonian pasture and forest. In: Gash, J.C.; Nobre, C.; Roberts, J.; Victoria, R. (Eds). Amazonian Deforestation and Climate, John Wiley, New York, p. 437-458.

Xiu, A.; Pleim, J. 2001. Development of a land surface model. Part I: Application in a mesoscale meteorological model. Journal of Applied Meteorology, 40: 192-209.

Xue, Y.; P. J. Sellers; J. L. Kinter; J. Shukla. 1991. A simplified biosphere model for global climate studies. Journal of Climate, 4(3): 345-36.

\section{RECEBIDO EM 18/06/03 \\ ACEITO EM 03/06/05}

\section{APÊNDICE}

\section{PRINCIPAIS EQUAÇÕES DO MODELO SSIB}

Existem sete equações prognósticas no modelo SSiB que podem ser consideradas como um subconjunto das equações originais do SiB. A equação prognóstica para a temperatura do dossel $T_{c}$ é:

$$
C_{c} \frac{\partial T_{c}}{\partial t}=R_{n c}-H_{c}-\lambda E_{c}
$$

em que $C_{c}$ é a capacidade efetiva de calor no dossel, $R_{n c}$ é o saldo de radiação na superfície e $H_{c}$ e $l E_{c}$ são dados por:

$$
H_{c}=\frac{2\left(T_{c}-T_{a}\right)}{r_{b}} \rho c_{c}
$$

$$
\lambda E_{c}=\left(e_{*}\left(T_{c}\right)-e_{a}\right) \frac{\rho c}{\gamma}\left[\frac{W_{c}}{r_{b}}+\frac{1-W_{c}}{r_{b}-r_{c}}\right]
$$

em que $T_{a}$ e $e_{a}$ são a temperatura e a pressão de vapor d'água no espaço do ar do dossel, respectivamente, $e_{*}\left(T_{C}\right.$ ) é a pressão de vapor de saturação à temperatura $T, W_{c}$ é a fração úmida do dossel, $r_{b}$ e $r_{c}$ são as resistências aerodinâmica e de superfície, respectivamente, $\gamma$ é o coeficiente psicrométrico e $\lambda$ o calor latente de vaporização. O saldo de radiação à superfície é dado pela soma dos termos $R_{n c} \mathrm{e} R_{n g s}$, que se referem às componentes do dossel e da superfície, respectivamente, definidas por:

$$
R_{n c}=F_{c}-2 \sigma_{S} T_{C}^{4} V_{C} \delta_{t}+\sigma_{S} T_{g s}^{4} V_{C} \delta_{t}
$$

$$
R_{n g s}=F_{g s}-\sigma_{s} T_{g s}^{4}+\sigma_{s} T^{4} V_{c} \delta_{t}
$$


e $F_{c}$ e $F_{g s}$ são as somas das radiações de ondas curtas e longas absorvidas pelo dossel e pela superfície, respectivamente, $\sigma_{s}$ éa constante de Stefan-Boltzmann, $T_{c}$ é a temperatura do dossel, $V_{c}$ é a fração da cobertura vegetal, $\delta_{t}$ é a transmitância da radiação infravermelha termal para o dossel, e $T_{g s}$ é a temperatura da superfície. A equação para a temperatura da superfície, $\mathrm{T}_{\mathrm{gs}}$ é:

$$
C_{g s} \frac{\partial T_{g s}}{\partial t}=R_{n g s}-H_{g s}-\lambda E_{g s}-2 \pi C_{g s} \frac{\left(T_{g s}-T_{d}\right)}{\hat{o}}
$$

sendo $\hat{o}$ o comprimento do dia, $C_{g s}$ a capacidade efetiva de calor no solo, $T_{d}$ a temperatura do solo profundo, $H_{g s}$ e $l E_{g s}$ são os fluxos de calor sensível e calor latente da superfície, definidos por:

$$
\begin{aligned}
H_{g s} & =\frac{T_{g s}-T_{a}}{r_{d}} \rho c_{p} \\
\lambda E_{g s} & =\left[f_{h} e_{*} T_{g s}-e_{a}\right] \frac{\rho c_{p}}{\gamma} \frac{1}{r_{\text {surf }}+r_{d}}
\end{aligned}
$$

sendo $f_{b}$ a umidade relativa do ar na superfície e $r_{d}$ a resistência aerodinâmica entre a superfície e o espaço do ar do dossel, e $\boldsymbol{r}_{\text {surf }}$ a resistência da superfície, definida por:

$$
r_{\text {surf }}=a_{S}\left(1-\mathrm{w}_{1}^{b s}\right)
$$

em que $\mathrm{a}_{\mathrm{s}}$ e $\mathrm{b}_{\mathrm{s}}$ são constantes e $\mathrm{w}_{1}$ a umidade na primeira camada do solo. A equação para a temperatura do solo profundo $\mathrm{T}_{\mathrm{d}}$ é:

$$
C_{g s} \frac{\partial T_{d}}{\partial \mathrm{t}}=2\left(R_{n g s}-H_{g s}-\lambda E_{g s}\right) / \sqrt{365 \pi}
$$

A equação governante para a água armazenada no dossel é:

$$
\frac{\partial M_{c}}{\partial t}=P_{c}-D_{c}-\frac{E_{w c}}{\rho_{w}}
$$

sendo $\mathrm{P}_{\mathrm{c}}$ a precipitação. $\mathrm{D}_{\mathrm{c}}$ a taxa de drenagem de água, $\rho_{\mathrm{w}}$ a densidade da água $\mathrm{e} \mathrm{E}_{\mathrm{wc}}$ a taxa de evaporação da porção úmida da vegetação (Sellers et al. 1986). Essa taxa de evaporação é definida por:

$$
\lambda E_{w c}=\frac{\left[e\left(T_{c}\right)-e_{a}\right]}{r_{b}} \frac{\rho c_{p}}{\gamma}
$$

As equações governantes para a umidade do solo $\mathrm{w}_{1}, \mathrm{w}_{2}$ e $\mathrm{w}_{3}$ nas três camadas são:

$$
\begin{aligned}
& \frac{\partial w_{1}}{\partial t}=\frac{1}{\Theta_{s} D_{1}}\left[P_{1}-Q_{12}-\frac{1}{\rho_{w}}\left(E_{g s}+b_{1} E_{d c}\right)\right] \\
& \frac{\partial w_{2}}{\partial t}=\frac{1}{\ominus_{s} D_{2}}\left[Q_{12}-Q_{23}-\frac{1}{\rho_{w}}\left(b_{2} E_{d c}\right)\right]
\end{aligned}
$$




$$
\frac{\partial w_{3}}{\partial t}=\frac{1}{\ominus_{s} D_{3}}\left[Q_{23}-Q_{3}\right]
$$

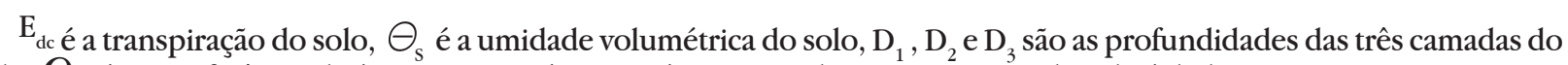
solo. $Q_{n j}$ é a transferência de água entre a n-ésima e j-ésima camadas. A evaporaçấo do solo é dada por:

$$
\lambda E_{d c}=\frac{\left[e\left(T_{c}\right)-e_{a}\right]}{r_{c}+r_{b}} \frac{\rho c_{p}}{\gamma}\left(1-w_{c}\right)
$$

em que $\mathrm{w}_{\mathrm{C}}$ é a fração de umidade do dossel. As temperaturas e a pressão de vapor d'água dentro do espaço do ar do dossel são determinadas pelas equações do balanço de energia:

$$
\begin{gathered}
H_{c}+H_{g s}=\frac{[T a-T r]}{r_{b}} \rho c_{p} \\
\lambda E_{c}+\lambda E_{g s}=\frac{\left[e_{a}-e_{r}\right]}{r_{a}} \frac{\rho c_{p}}{\gamma}
\end{gathered}
$$

